\title{
¿POSTUREO O DISFRUTE DE LA EXPERIENCIA GASTRONÓMICA? EFECTOS DE LA REPUTACIÓN Y LA EXPERIENCIA EN LA RECOMENDACIÓN DE LOS RESTAURANTES DE ALTO NIVEL*
}

\author{
Natalia Daries Ramón \\ Berta Ferrer Rosell \\ Eduard Cristóbal Fransi \\ Estela Mariné Roig \\ Universitat de Lleida
}

\section{RESUMEN}

En este estudio se ha desarrollado un modelo explicativo basado en la teoría de la motivación fundamentada por la posibilidad de obtener reputación y reconocimiento por consumir gastronomía de alto nivel y la motivación basada en el disfrute de la experiencia gastronómica, con la finalidad de analizar sus efectos en la intención de recomendación de este tipo de experiencias. El estudio confirma que la experiencia gastronómica y el estatus afectan positivamente a la intención de revisita y a la recomendación del consumo de gastronomía de alto nivel, siendo el efecto de la experiencia mucho más fuerte.

Palabras clave: Turismo gastronómico; Ecuaciones estructurales; Estatus; Recomendación; Restaurantes Michelin.

Fecha de recepción: 25 de septiembre de 2017.

Fecha de aceptación: 18 de abril de 2018.

Departamento de Administración de Empresas. Universidad de Lleida. C/ Jaume II, 73. Campus de Cappont. 25001 LLEIDA (España).E-mail: ndaries@aegern.udl.cat, berta.ferrer@aegern.udl.cat, ecristobal@aegern.udl. cat, estela.marine@aegern.udl.cat

* Agradecimientos: Los autores agradecen a la Generalitat de Catalunya por la acreditación concedida como Grupo Emergente de Investigación GRTESC (2014 SGR 82). A su vez agradecen a la Universidad de Lleida, por la ayuda concedida dentro del marco del Programa de Promoción de la Investigación de la Universitat de Lleida, convocatoria 2017-TR265, y también al Ministerio de Economía, Industria y Competitividad por la concesión del proyecto TURCOLAB (ECO2017-88984-R). 


\title{
Posturing or enjoying the gastronomic experience? Effects of reputation and experience in the recommendation of upscale restaurants
}

\begin{abstract}
This study has developed an explanatory model based on the theory of motivation related to the possibility of obtaining reputation and recognition for consuming high-end gastronomy and to the enjoyment of the gastronomic experience, with the purpose of analyzing their effects on the intentions to return and recommend this type experience. The study confirms that the gastronomic experience and the status positively affect the intentions of returning and recommending the consumption of high-end gastronomy, being the effect of the experience much stronger.
\end{abstract}

Keywords: Gastronomy Tourism; Structural Equations; Status; Recommendation; Michelin Restaurants.

\section{INTRODUCCIÓN}

En los últimos años, el aumento de la importancia de la gastronomía, propiciada tanto por los medios de comunicación de masas como por la participación generalizada de la población en las redes sociales, ha permitido la expansión de un tipo particular de consumo, el denominado consumo de gastronomía de alto nivel. Estos restaurantes exclusivos y elegantes están diseñados para proporcionar comida, servicio y un ambiente de calidad superior (Hyun y Kang 2014; Daries et al., 2018).

Sin embargo, con el auge de las redes sociales, ayudado por las tecnologías, han surgido nuevas prácticas sociales relacionadas a la formación de la identidad personal en línea y a la forma de mostrarla a los demás (Livingstone, 2008; Wakefield y Wakefield, 2016). Es en este escenario donde numerosos autores apuntan que lo más importante para muchos usuarios es la imagen que se comparte, el estatus, la notoriedad y buscar la aprobación de los demás (Carsten et al. 2010); o en palabras coloquiales lo que ha venido a llamarse el "postureo" en línea. En el contexto de nuestro trabajo, que es el consumo de la gastronomía de alto nivel, es difícil discernir si el consumidor realmente está disfrutando de su experiencia culinaria y gastronómica o es la comida en sí la que se vuelve parte del decorado de un estilo de vida ligado al estatus y la aprobación social (Goolaup et al., 2017).

Thompson y Prideaux (2009) apuntan a que los consumidores de gastronomía de alto nivel valoran la experiencia global, ya que sus preferencias varían respecto a otros tipos de consumidores, pasando a valorar la calidad en lugar de la cantidad, y priorizando la originalidad, la creatividad, la puesta en escena, el descubrimiento de nuevos sabores, texturas, etc. Por ello, García Henche (2017), Daries et all. (2017), afirman que los turistas, en la actualidad, consideran las experiencias gastronómicas como un elemento clave a la hora de elegir el destino. 
Aun así, existen pocos estudios que expliquen las motivaciones o actitudes referidas al consumo de este tipo de gastronomía. Por ello, en este estudio pretendemos identificar las motivaciones que impulsan al consumo de la restauración de alto nivel determinando la relación explicativa entre el disfrute de la experiencia gastronómica en sí y la reputación o estatus que le proporciona dicha experiencia en las recomendaciones o en la intención de vuelta. Por tanto, el objetivo de este estudio es saber si el público que recomienda y es asiduo a este tipo de establecimientos realmente está interesado en la gastronomía, y disfruta de los sabores, nuevas propuestas, etc., o realmente visita este tipo de restaurantes para poder contarlo a sus amigos o compartirlo en las redes sociales.

Para ello se ha desarrollado un modelo explicativo basado en la teoría de la motivación de ecuaciones estructurales en el que se representan los elementos que contribuyen a la intención de vuelta y recomendación: la reputación, referida al estatus o prestigio que le proporciona a una persona el consumo de gastronomía de alto nivel centrada en el caso concreto de los restaurantes con estrella Michelin y la satisfacción entendida como el disfrute y la diversión relacionada con la experiencia gastronómica.

Por último, aportaremos en la discusión sobre los resultados, recomendaciones a los administradores de este tipo de restaurantes, las principales limitaciones y las futuras líneas de investigación a partir de los resultados del presente estudio.

\section{APROXIMACIÓN TEÓRICA A LOS CONSTRUCTOS}

La literatura científica respalda que la gastronomía de alto nivel establece sinergias con motivaciones diferentes. Autores como Fodness (1994), Hall y Winchester (2001), Pollard et al. (2002), o Yang y Mattila (2014; 2017) fundamentan que una de las principales razones que conlleva a la gente a acudir a restaurantes de buena gastronomía o de alto nivel es la reputación o estatus que les proporciona. Por otro lado podemos encontrar otra línea de investigación (Cohen y Avieli, 2004; Goolaup et al., 2017; Gyimòthy y Mykletun, 2009; Hjalager, 2004, Ignatov y Smith, 2006; Long 2004; Otis 1984; Rust y Oliver, 2000 o Wolf et al., 2004), en donde se argumenta que la razón principal del consumo de este tipo de gastronomía es la generación de buenas experiencias. A su vez hay autores que afirman que en la decisión de consumo confluyen las dos razones (Fields 2002; Hyun y Park, 2016; Kim et al., 2009, Kivela y Crotts, 2006; McIntosh et al., 1995).

Según Kim, Eves y Scarles (2009) en un estudio realizado a consumidores del Reino Unido sobre las motivaciones de comer en buenos restaurantes durante las vacaciones, llegaron a la conclusión de que los principales factores motivacionales son aquellos relacionados con la experiencia: experiencias excitantes, escapar de la rutina, experiencias auténticas, conocer experiencias sensoriales nuevas y por otra parte los factores relacionados con el prestigio o estatus que ofrecen estas experiencias. Finalmente comentar que diversos estudios encontraron que una buena experiencia puede mejorar la actitud del turista hacia el destino (Lee y Moscardo, 2005), su satisfacción (Chen y Chen 2010) y su comportamiento (Tsai y Lu, 2012). Por lo tanto, sabiendo de la importancia del valor de la experiencia vivida mientras se visita un lugar, la imagen gastronómica del destino turístico debe ser potenciada (Tsai y Wang, 2017). 


\subsection{Experiencia gastronómica}

La experiencia gastronómica la podemos enmarcar dentro del turismo experiencial. No es lo mismo visitar un destino que sentir un destino (olores, colores, gusto, texturas...) y vivir un destino con sus habitantes. Hay que integrarse en la forma de vivir y en la cultura propia de cada lugar para crear un recuerdo que perviva después del viaje y que pueda ser compartido con los demás (Carballo et al., 2015; García Henche, 2017).

Se puede definir el Turismo Experiencial como una modalidad turística en la que los visitantes no visitan los recursos turísticos tradicionales, sino que buscan otras atracciones con el objetivo de experimentar sensaciones memorables. Se trata de una forma de turismo en la que los destinos no se eligen por sus estándares turísticos sino a partir de una idea o experimento previo (Suau, 2012). En su trabajo Kim et al. (2012) diseñan una escala con siete dimensiones para valorar las experiencias memorables: hedonismo, refrescante, cultura local, sentido, conocimiento, implicación y novedad. Siguiendo esta línea, Tung y Ritchie (2011) identificaron por su lado cuatro dimensiones principales que integran estas experiencias memorables: las expectativas, las emociones, las consecuencias derivadas de la experiencia y la recolección, o sea, las actuaciones posteriores al viaje para recordarlo, revivirlo, etc. en el destino de origen de los turistas. El turismo gastronómico es un importante valor para generar estas experiencias memorables (Rivera, 2013).

Long (2004) define turismo culinario como aquel turismo que está planteado con el deseo de disfrutar experiencias gastronómicas diferentes que pueden ir desde comidas totalmente exóticas a aquellas más tradicionales. La prioridad de este tipo de turismo es degustar los platos únicos y exclusivos de cada lugar que forman parte del conocimiento local y de la cultura integral (Wolf et al., 2004; Ignatov y Smith, 2006; Jiménez Beltrán et al., 2016). Es decir, representa mucho más que el mero hecho de consumir comida en un viaje.

Kim et al. (2009) en su estudio sobre las motivaciones de consumir gastronomía durante los viajes y las vacaciones, comentan que el hecho de comer comida de calidad que no han probado anteriormente y recomendada por las guías turísticas, provoca que la experiencia sea excitante. Otis (1984) relata que comer en buenos restaurantes se convierte en una actividad más dentro de las vacaciones en la que se disfruta igual o más que cualquier otra, incluso que provoca grandes expectativas y sentimientos de felicidad. Por su lado, Rust y Oliver (2000) comentan que una buena experiencia gastronómica mejora la vida de la gente y que si esa experiencia supera las expectativas creadas se recordará por mucho tiempo. Sparks et al. (2003) y Gyimòthy y Mykletun, (2009) asocian la buena gastronomía con la búsqueda de experiencias culinarias cada vez más importantes como son nuevos sabores, texturas diferentes y nuevas sensaciones que no se pueden vivir cada día de tu vida. Fields (2002) destaca que se trata de una experiencia física con percepciones sensoriales a través de la vista, el gusto y el olfato. Goolaup et al., (2017), definen la gastronomía en destino como una experiencia de viaje extraordinaria y la relacionan con el contexto social del turista. Finalmente, Kivela y Crotts (2006) definen la experiencia gastronómica como una experiencia sensorial placentera que ofrece un factor de placer lo suficientemente intenso como para atraer a 
los turistas a un destino. De hecho, el turismo gastronómico es visto como una de las grandes apuestas para potenciar o consolidar destinos turísticos debido a la creciente motivación de los turistas a conocer la cultura gastronómica del lugar (Jiménez Beltrán et al., 2016). Millán et al. (2016) definen el turismo gastronómico básicamente como una experiencia. Una experiencia directa en el territorio de producción y donde las experiencias sensoriales y culturales que la gastronomía ofrece se convierten en nuevos elementos que se pueden añadir al descubrimiento de paisajes nuevos y desconocidos por turistas (Medina, 2015).

\subsection{Reputación}

Cada vez más frecuentemente, la literatura sobre turismo gastronómico destaca el importante papel de la reputación y el prestigio que se les atribuye a los turistas que realizan este tipo de actividad. Al hecho de comer fuera se le han atribuido "connotaciones de clase" (Warde y Martens, 2000). Estos autores afirman que la distinción de clases se manifiesta a menudo a través de patrones diferenciados de consumo y diferentes tipos de lugares para comer. El consumo de alimentos en el turismo también implica un cierto grado de distinción social, así como un consumo notorio (Chang et al., 2010).

Las motivaciones gastronómicas de estatus y prestigio no son ajenas a la existencia de restaurantes de alto nivel. Estos restaurantes buscan transmitir una imagen de calidad y exclusividad para transmitir un elevado nivel de estatus y prestigio al comensal que lo frecuente (Oliveira, 2011). Fields (2002), presenta un interesante modelo sobre los turistas gastronómicos, desde el punto de vista motivacional, basado en el modelo diseñado por Mcintosh et al. (1995). El modelo de Fields (2002) es aplicable a este tipo turistas y asume que siendo la gastronomía el motivo del desplazamiento, existen otras motivaciones de orden físico, cultural, interpersonal y de estatus y prestigio asociadas al viaje. Por tanto, Fields (2002) resalta una motivación generalizada de este tipo de turistas que buscan el reconocimiento y aprobación de los demás. Siguiendo esta línea Kim et al. (2009), elaboran un modelo de consumo en restaurantes locales por parte de los turistas basado en tres dimensiones. La primera de ellas contiene factores de motivación que incluyen aspectos como la búsqueda de la experiencia, el prestigio social y la autenticidad. La segunda dimensión incluye factores demográficos y engloba, entre otros, el género, la edad y el nivel educativo. Y el tercer elemento se basa en los factores psicológicos.

Mak et al. (2013), analizan los factores motivacionales del consumo de productos gastronómicos por parte de los turistas en Hong Kong. Ellos extraen 14 factores, categorizados en cinco dimensiones (simbólico, obligatorio, contraste, extensión y placer). De la primera dimensión, "simbólico", se destaca el factor de "Prestigio". Chang et al. (2010) por su lado sugieren que los turistas están motivados para participar en experiencias gastronómicas especiales y memorables como son las que ofrecen los restaurantes de alto nivel, para posteriormente compartirlas con sus amigos cuando regresan a casa.

Fodness (1994) argumenta que la experiencia de comer en restaurantes de lujo y disfrutar de vacaciones gastronómicas proporciona un estatus que impresiona a la gente. McIntosh et al. (1995), a su vez, los relaciona con factores motivacionales de estatus y 
prestigio considerando que estos aumentan la autoestima, el reconocimiento y el deseo de llamar la atención de los demás. Hall y Winchester (2001) demuestran en su trabajo que los individuos que tienen grandes conocimientos sobre enología y explican sus experiencias acerca de los buenos restaurantes que han acudido, tienen influencia en la gente y proporcionan impresiones favorables. Pollard et al. (2002) manifiestan que ir a restaurantes de nouvelle cuisine o con Estrella Michelin se asocia a la gente con un estatus elevado. Finalmente, Yang y Mattila (2017) investigan el efecto combinado de la necesidad de estatus del consumidor y del tipo de producto sobre las intenciones de recomendación. Los resultados sugerían que los clientes de alta necesidad de estatus, son más propensos a comentar su experiencia que los comensales de baja necesidad de estatus.

Hjalager (2004) basándose en las investigaciones de Cohen (1984) categoriza a los turistas gastronómicos en cuatro clases basándose en su estilo de vida: el recreativo, el festivo, el existencial y el experimental. Los turistas recreativos y festivos son aquellos más tradicionales que no les interesa conocer o probar alimentos nuevos. Los recreativos se cocinan ellos mismos en sus apartamentos con productos similares a los de su país que compran en supermercados. Los festivos comen en restaurantes internacionales donde priorizan la cantidad en vez de la calidad. El turista existencial es aquel que le gusta probar alimentos del país y comer en restaurantes locales no muy caros donde no van los turistas. Y por último se encuentra el turista experimental que es aquel en el que el disfrute de la experiencia del consumo de comida y bebida en vacaciones es parte de su estatus y personalidad. Para estos últimos, priorizan la calidad, ponen en valor los restaurantes de moda y los chefs prestigiosos. Asimismo, comer fuera conlleva arreglarse con ropa de moda de diseño, llevar buenos coches y estar al corriente de las últimas tendencias gastronómicas. Por tanto, sus suvenires son botellas de vino de alta calidad y libros de cocineros de los restaurantes que han visitado.

Así pues, podemos considerar que la importancia de la reputación y la experiencia en el consumo de la gastronomía de alto nivel están relacionadas, y estos dos constructos se comportan similarmente.

\subsection{Intención de revisita y recomendación}

En la industria turística hay numerosos estudios que evidencian la relación existente entre la satisfacción y la intención de vuelta, de revisitar el lugar y de recomendarlo a otra gente (Eid, 2015; Kozak, 2001; Kozak y Rimmington, 2000; Yoon y Uysal, 2005). De hecho la satisfacción con la experiencia turística se considera por la mayoría de autores el motivo más directo que influencia la intención de vuelta, la recomendación a otros y la lealtad (Antón et al., 2017; Björk y Kauppinen-Räisänen, 2017; Gonçalves et al., 2016).

Los turistas satisfechos son grandes prescriptores considerados mucho más efectivos que cualquier medio de comunicación o estrategia de marketing (Yoon y Uysal, 2005). Antón et al. (2017), consideran que tanto la experiencia relacionada con elementos del destino como es la gastronomía, así como la obtención de prestigio, entre otros, son motivos que atraen a los turistas a los destinos y que determinan su satisfacción y lealtad a los mismos. Por otro lado, experiencias positivas y placenteras relacionadas con 
la gastronomía afectan positivamente a la intención del viajero de volver a visitar un destino (Kim et al., 2011; Quan y Wang, 2004). En un estudio reciente, Jiménez Beltrán et al. (2016) encontraron que las variables que más influencian la satisfacción con la experiencia gastronómica son la calidad de los platos, la innovación y los nuevos sabores, en detrimento de otros elementos como las instalaciones o la atmósfera del establecimiento. Además, participar en el consumo de gastronomía de alto nivel es una oportunidad para ganar reconocimiento y reputación en la era digital (Zheng y Gretzel, 2010) ya que la visita a restaurantes de alto nivel es una buena oportunidad para compartir información en redes sociales. Lin (2014) elabora un modelo para analizar la intención de revisita de los balnearios, y señala la experiencia culinaria como uno de los factores más significativos. Por otro lado en un estudio realizado por Basri et al., (2016), señalan que la calidad del servicio, la calidad de los alimentos, la calidad del entorno físico y el precio son los atributos más relevantes para la toma de decisiones de visita a restaurantes de alto nivel de Malasia, la revisita y la recomendación a través del Word of Mouth.

Adam et al. (2015), llevaron a cabo una investigación dirigida a 768 turistas internacionales que habían acudido a restaurantes de lujo en Ghana. En su trabajo mostraban que la satisfacción con los factores relacionados con los aspectos tangibles, la fiabilidad, la seguridad-empatía y la capacidad de respuesta, tenía una relación directa con la intención de revisita.

Kivela y Crotts realizaron un estudio en 2006 con más de 1.200 encuestas a turistas no residentes, en restaurantes selectos de dos localidades turísticas en Hong Kong llegando a la conclusión de que la gastronomía de calidad era un constructo válido para ser utilizado como herramienta de marketing, evidenciando que las experiencias gastronómicas favorables influyen de forma positiva en la intención de vuelta contribuyendo a la creación de experiencias de viaje y catalogándolos como de alta calidad. Pollard et al. (2002) y Fields (2002) manifiestan a su vez que la lealtad de los turistas está claramente asociada con las experiencias positivas.

\section{FORMULACIÓN DE HIPÓTESIS}

Consecuentemente teniendo en cuenta los argumentos previos que relacionan la experiencia gastronómica y la reputación con la intención de recomendación podemos desarrollar las siguientes hipótesis:

- H1 El grado de reputación percibida de participar en el consumo de restaurantes de alto nivel influye positivamente en las intenciones de vuelta y recomendación. (A más reputación percibida, más altas las intenciones de vuelta y de recomendación).

- H2 El grado de la experiencia percibida de participar en el consumo de restaurantes de alto nivel influye positivamente en las intenciones de vuelta y recomendación. (A más experiencia percibida, más altas las intenciones de vuelta y de recomendación).

El objetivo del modelo hipotético propuesto es conocer cuáles son los efectos de la reputación y la experiencia en la intención de vuelta y la recomendación de la gastronomía de alto nivel. 


\section{Figura 1 MODELO TEÓRICO}

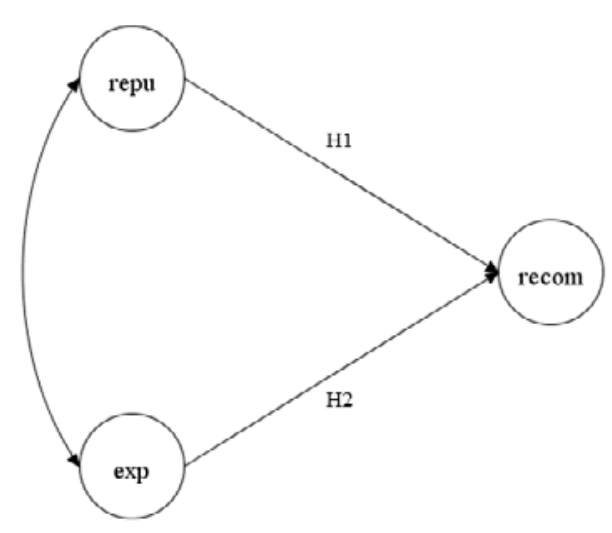

Fuente: Autores (2017)

Leyenda: recom=Recomendación; repu=Reputación; exp=Experiencia

\section{DATOS Y MÉTODO}

\subsection{Escalas de medición, diseño del estudio y método de análisis}

Para realizar el estudio se diseñó una investigación causal usando una encuesta de muestra transversal. El cuestionario consistió en las siguientes secciones principales: preguntas que midieron los siguientes constructos: la reputación referida al estatus aportado por este tipo de consumo, la satisfacción percibida como experiencia gastronómica, la recomendación o intención de vuelta y las preguntas diseñadas para recopilar la información demográfica y el comportamiento de los consumidores de gastronomía de alto nivel.

Basándonos en literatura previa y adaptándolo para el caso del consumo de gastronomía de alto nivel se desarrollaron escalas tipo Likert de 5 puntos para la medición de las variables. Concretamente para la variable disfrute de la experiencia gastronómica se han utilizado los trabajos de Quan, y Wang (2004) y Van der Heijiden (2004). Para la variable basada en reputación y prestigio, se ha basado en los trabajos de Foodness (1994) y Kim et al. (2009) y finalmente para la recomendación, se han consultado los trabajos de Chi et al. (2008), Boo et al. (2009) y Kim et al. (2010).

La figura 2 muestra el modelo de medida junto con la especificación del modelo teórico.

La población objeto de estudio se centró en los individuos que habían comido en un restaurante estrella Michelin español en los dos últimos años. Tal como se observa en la Tabla 1 la muestra fue seleccionada a través de grupos de Facebook sobre gastronomía y viajes, y el Facebook de blogueros gastronómicos de España de reconocido prestigio y con un gran número de seguidores, etc. 


\section{Figura 2 \\ MODELO DE MEDIDA Y MODELO TEÓRICO DE ESTUDIO}

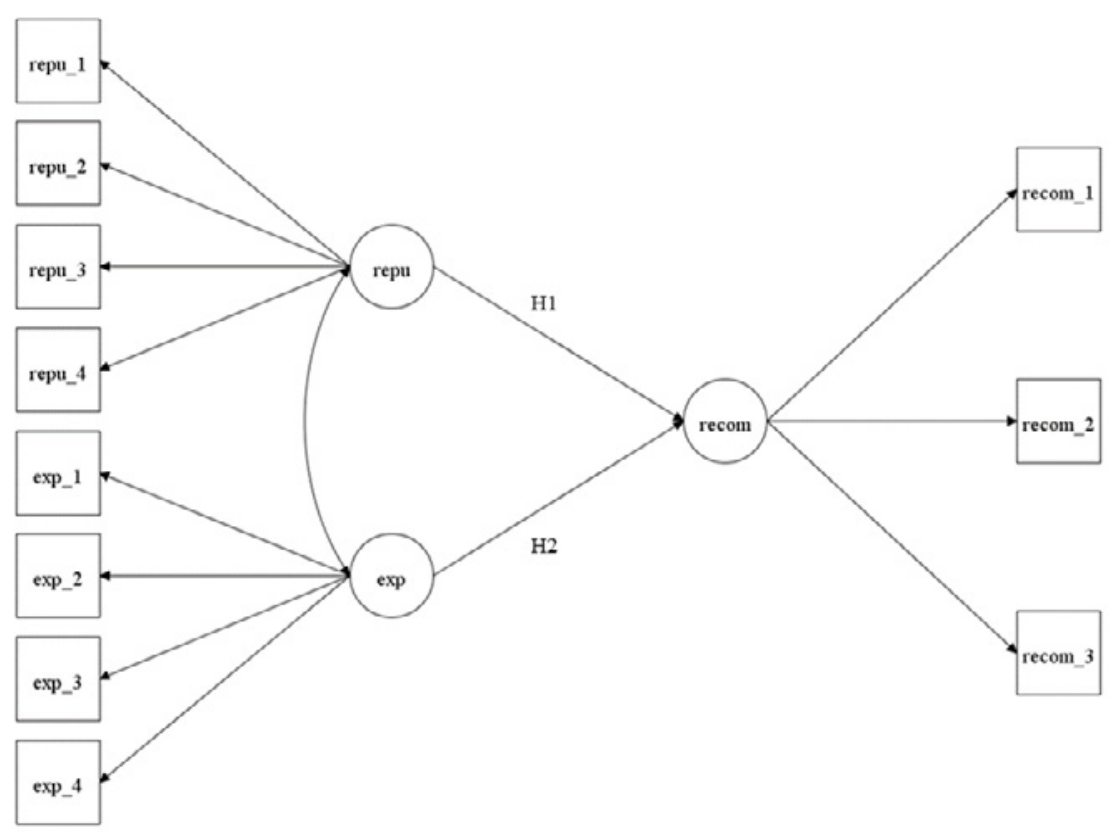

Tabla 1

PLATAFORMAS UTILIZADAS PARA ADMINISTRAR LA ENCUESTA

\begin{tabular}{|l|c|}
\hline Página de Facebook de blogueros de cocina & Seguidores \\
\hline Webosfritos & 96.918 \\
\hline Garbancita & 11.798 \\
\hline Grupos relacionados con la gastronomía & \\
\hline Gastroturismo o Turismo Gastronómico & 497 \\
\hline Gastronomic excellence reputación gastronómico & 4207 \\
\hline Gastronomías del Mundo & 13.642 \\
\hline Gastrotip Turismo Gastronómico por España & 280 \\
\hline Grupos relacionados con viajes & \\
\hline España se come & 4867 \\
\hline Viajeros & 2837 \\
\hline
\end{tabular}

Fuente: Autores (2017).

La recogida de datos se realizó de julio a diciembre 2016, a través de un cuestionario auto-administrado online. Se envió el enlace de la encuesta a los blogueros y a los administradores de los grupos, relacionados con la gastronomía y viajes de las páginas de Facebook especializadas, para que lo colgaran a sus seguidores (solo los seguidores 
de los blogs y amigos de la página de Facebook tenían acceso). Se recogieron un total de 1500 encuestas de las cuales 432 se consideraron respuestas válidas, lo que da un error muestral de $4,8 \%$ (considerando una población infinita), para un nivel de confianza del $95 \%$ y $p=q=50 \%$. La encuesta fue validada por un experto académico en metodología de encuestas y un experto académico en gastronomía. La prueba piloto consistió en la realización de la encuesta a 25 expertos gastronómicos y del sector, cocineros de restaurantes estrellas Michelin, sumilleres de reconocido prestigio y consumidores habituales de restaurantes de alto nivel. Se realizaron las modificaciones pertinentes en la disposición de diferentes preguntas, se mejoró la redacción y se verificó el correcto entendimiento de cada pregunta.

El análisis del modelo propuesto se ha realizado a través de modelos de ecuaciones estructurales, que permite testear relaciones causales entre variables dependientes e independientes de forma simultánea. Se ha utilizado el software estadístico MPlus7.4, y la técnica de máxima verosimilitud robusta $(M L R)$, ya que se han tenido en cuenta los valores faltantes en algunas variables.

\subsection{Datos}

La muestra está compuesta por el $64 \%$ de mujeres y el $36 \%$ de hombres. Por edades, el $21 \%$ de los encuestados tiene hasta 34 años, el $68 \%$ tiene entre 35 y 54 años, y el 11\% tiene más de 55 años. En referencia al nivel de estudios de los encuestados, el 86,5\% tiene estudios universitarios y el resto $(13,5 \%)$ tiene hasta educación secundaria. Además, el $26,6 \%$ tiene unos ingresos mensuales netos de su hogar de hasta $2.000 €$, el $59 \%$ tiene unos ingresos de entre 2.001 y $5.000 €$ y el 14,4\% ingresa más de $5.001 €$.

Antes de contrastar el modelo teórico propuesto, es conveniente realizar el análisis descriptivo de las respuestas obtenidas de las variables objeto de estudio.

Tabla 2

\section{VARIABLES UTILIZADAS Y DESCRIPTIVOS}

\begin{tabular}{|l|l|c|c|c|c|c|}
\hline Constructos & Ítems & $\mathbf{n}$ & Media & Mín. & Máx. & $\begin{array}{c}\text { Desv. } \\
\text { Estánd. }\end{array}$ \\
\hline & $\begin{array}{l}\text { Repu_1: } \\
\text { El estatus que me proporciona }\end{array}$ & 428 & 1,98 & 1 & 5 & 1,35 \\
\cline { 2 - 6 } & $\begin{array}{l}\text { Repu_2: } \\
\text { Visitar Restaurantes Michelin } \\
\text { me ayuda a ser aceptado por } \\
\text { otras personas }\end{array}$ & 427 & 1,52 & 1 & 5 & 1,10 \\
\cline { 2 - 7 } $\begin{array}{l}\text { Estatus/ } \\
\text { Reputación }\end{array}$ & $\begin{array}{l}\text { Repu_3: } \\
\text { Visitar restaurantes Michelin } \\
\text { impresiona a la gente }\end{array}$ & 425 & 1,81 & 1 & 5 & 1,26 \\
\cline { 2 - 7 } & $\begin{array}{l}\text { Repu_4: } \\
\text { Poder contarlo más tarde a } \\
\text { mis familiares y amigos }\end{array}$ & 427 & 2,11 & 1 & 5 & 1,32 \\
\hline
\end{tabular}




\begin{tabular}{|c|c|c|c|c|c|c|}
\hline Constructos & Ítems & $\mathbf{n}$ & Media & Mín. & Máx. & $\begin{array}{l}\text { Desv. } \\
\text { Estánd. }\end{array}$ \\
\hline \multirow{4}{*}{ Experiencia } & $\begin{array}{l}\text { Exp_1: } \\
\text { He disfrutado mucho }\end{array}$ & 431 & 4,61 & 1 & 5 & 0,75 \\
\hline & $\begin{array}{l}\text { Exp_2: } \\
\text { Mi elección fue acertada }\end{array}$ & 425 & 4,57 & 1 & 5 & 0,80 \\
\hline & $\begin{array}{l}\text { Exp_3: } \\
\text { Estoy satisfecho con mi } \\
\text { experiencia }\end{array}$ & 431 & 4,57 & 1 & 5 & 0,82 \\
\hline & $\begin{array}{l}\text { Exp_4: } \\
\text { Es exactamente lo que } \\
\text { necesitaba }\end{array}$ & 430 & 4,43 & 1 & 5 & 0,94 \\
\hline \multirow{3}{*}{$\begin{array}{l}\text { Revisita/ } \\
\text { Recomendación }\end{array}$} & $\begin{array}{l}\text { Recom_1: } \\
\text { Intentaré volver a este } \\
\text { restaurante en los próximos } \\
\text { años }\end{array}$ & 424 & 3,66 & 1 & 5 & 1,46 \\
\hline & $\begin{array}{l}\text { Recom_2: } \\
\text { Animaré a mis familiares } \\
\text { y amigos a que visiten el } \\
\text { restaurante }\end{array}$ & 427 & 4,01 & 1 & 5 & 1,24 \\
\hline & $\begin{array}{l}\text { Recom_3: } \\
\text { Recomendaría este restaurante } \\
\text { si alguien me pidiera consejo }\end{array}$ & 429 & 4,43 & 1 & 5 & 1,07 \\
\hline
\end{tabular}

Fuente: Autores (2017).

En la Tabla 2 se observa que la reputación no es lo que los individuos buscan cuando comen en restaurantes Michelin. Las medias de las variables no llegan a 2 sobre 5. En cambio, cuando se les pregunta sobre la experiencia gastronómica, en general, consideran que es una buena experiencia (medias entre 4,4 y 4,6). Finalmente, sobre la intención de recomendar el restaurante, también se observa que las medias son altas, y los encuestados lo recomendarían y animarían a amigos y familiares.

\section{ANÁLISIS Y RESULTADOS}

\subsection{Evaluación del modelo de medida}

La tabla 3 muestra las cargas factoriales estandarizadas de cada ítem sobre su constructo y su correspondiente estadístico $t$, la fiabilidad de medida de cada constructo mediante el Alfa de Crombach y la varianza media extraída (AVE). Según se observa, el modelo de medida es fiable, ya que las cargas factoriales son altas (superiores a 0,71), lo cual según Chin (1998), indica que al menos el 50\% de la varianza de los factores se ve reflejada en el ítem observable. Por otro lado, la varianza media explicada (AVE) de cada factor es superior a 0,5, lo que según Fornell y Larcker (1981) indica que se explica el $50 \%$ o más de la varianza del indicador, y los valores de Alfa de Crombach son también superiores a 0,7 . 
Tabla 3

CARGAS FACTORIALES, ESTADÍSTICO T, ALFA DE CROMBACH Y VARIANZA MEDIA EXPLICADA (AVE)

\begin{tabular}{|c|c|c|c|c|c|}
\hline Constructos & Ítems & $\begin{array}{c}\text { Cargas factoriales } \\
\text { estandarizadas }\end{array}$ & Estadístico $t$ & $\begin{array}{c}\text { Alfa } \\
\text { Crombach }\end{array}$ & AVE \\
\hline \multirow{4}{*}{ Reputación } & Repu_1 & 0,82 & 29,48 & \multirow{4}{*}{0,89} & \multirow{4}{*}{0,67} \\
\hline & Repu_2 & 0,86 & 34,14 & & \\
\hline & Repu_3 & 0,85 & 26,14 & & \\
\hline & Repu_4 & 0,78 & 24,22 & & \\
\hline \multirow{4}{*}{ Experiencia } & Exp_1 & 0,94 & 70,72 & \multirow{4}{*}{0,96} & \multirow{4}{*}{0,87} \\
\hline & Exp_2 & 0,95 & 55,34 & & \\
\hline & Exp_3: & 0,96 & 85,71 & & \\
\hline & Exp_4: & 0,89 & 32,72 & & \\
\hline \multirow{3}{*}{ Recomendación } & Recom_1 & 0,72 & 23,95 & \multirow{3}{*}{0,88} & \multirow{3}{*}{0,71} \\
\hline & Recom_2 & 0,87 & 40,18 & & \\
\hline & Recom_3 & 0,92 & 51,90 & & \\
\hline
\end{tabular}

Fuente: Autores (2017)

\subsection{Evaluación del modelo teórico}

El modelo resultante es estadísticamente significativo, con un Chi cuadrado de 108,195 (41 grados de libertad, $\mathrm{p}<0,000$ ). Otros indicadores de ajuste global del modelo tradicionalmente usados se encuentran dentro de los valores recomendados por la literatura: RMSEA: 0,062, CFI: 0,969, TLI: 0,958, por lo que el modelo ajusta satisfactoriamente (Hu y Bentler, 1999).

\section{Tabla 4}

\section{RESULTADOS DEL MODELO TEÓRICO}

\begin{tabular}{|c|c|c|c|c|}
\hline & $\begin{array}{l}\text { Coeficiente }(\boldsymbol{\beta}) \\
\text { estandarizado }\end{array}$ & Estadístico $t$ & Significación & $\begin{array}{l}\text { Resultado } \\
\text { Hipótesis }\end{array}$ \\
\hline $\begin{array}{l}\text { H1: Reputación -> } \\
\text { Recomendación (+) }\end{array}$ & 0,096 & 3,981 & $\mathrm{P}<0,000$ & Soportada \\
\hline $\begin{array}{l}\text { H2: Experiencia -> } \\
\text { Recomendación }(+)\end{array}$ & 0,838 & 27,615 & $\mathrm{P}<0,000$ & Soportada \\
\hline
\end{tabular}

Fuente: Autores (2017)

Tal y como se observa en la Figura 3 y en la Tabla 4 las dos hipótesis planteadas están soportadas por los coeficientes (estadísticamente significativos) de cada una de las relaciones. Así pues, damos respuesta al objetivo del estudio, que era analizar cómo la reputación que otorga consumir un restaurante de alto nivel y la experiencia gastronómica, afectan positivamente a la recomendación de consumir este tipo de restaurantes. 


\section{Figura 3 \\ EVALUACIÓN DEL MODELO DE MEDIDA Y DEL MODELO TEÓRICO}

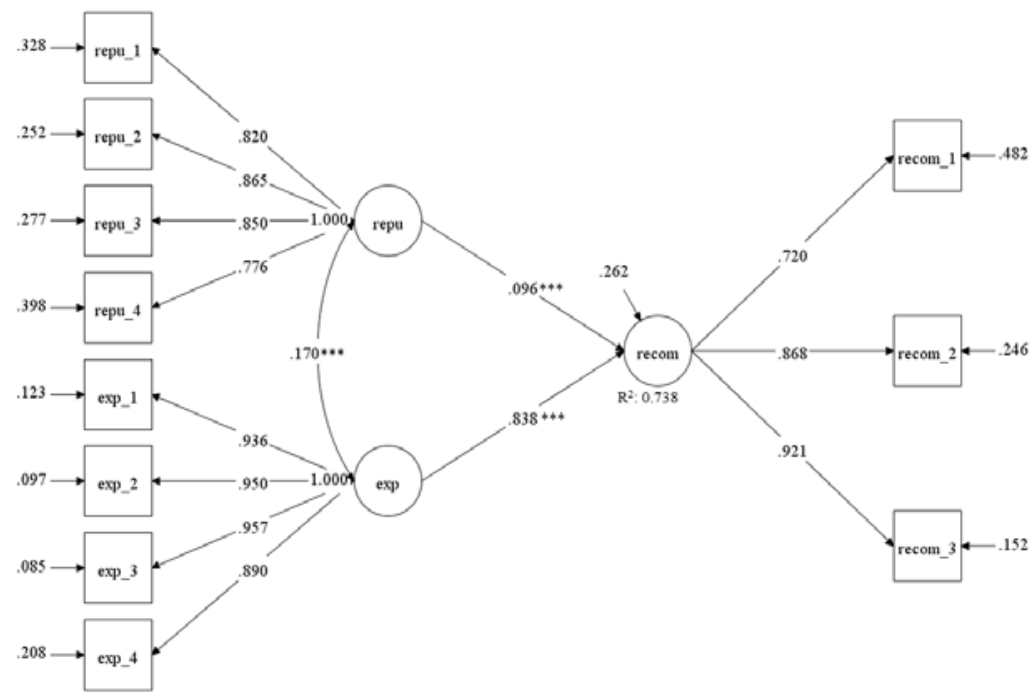

Fuente: Autores (2017)

$* * *$ p-value $<0,000$

El modelo también confirma que los constructos reputación y experiencia covarían, aunque sea poco (covarianza de 0,170, estadísticamente significativa). Esto significa que se relacionan y se comportan de forma similar, de acuerdo con lo apuntado por los autores Kivela y Crotts, (2006); McIntosh et al., (1995) y Kim et al. (2009) entre otros.

Por otro lado, se ha encontrado una relación mucho más fuerte entre la experiencia y la recomendación (0.838) que entre la reputación y la recomendación (0.096). De acuerdo con las afirmaciones de Pollard et al. (2002) y Fields (2002).

\section{CONCLUSIONES}

Este estudio analiza las motivaciones que afectan al consumo de gastronomía de gran nivel. Se propone un modelo de ecuaciones estructurales en el que se relacionan las motivaciones centradas en la experiencia y la reputación. Para ello nos hemos basado en trabajos previos como el de Kim et al. (2009) que relacionan la experiencia y la reputación y en el que se ha incorporado la recomendación. Este es un enfoque muy utilizado en destinos turísticos y que en este caso se ha adaptado a la gastronomía de alto nivel.

Los resultados del estudio muestran que ambas hipótesis iniciales se confirman: Por una parte el grado de reputación percibida de participar en el consumo de restaurantes de alto nivel influye positivamente en las intenciones de vuelta y recomendación. Y por otra parte, el grado de la experiencia percibida de participar en el consumo de restaurantes de alto nivel influye positivamente en las intenciones de vuelta y recomendación. 
Además, se ha encontrado que los constructos reputación y experiencia covarían o se comportan de forma similar en cierta medida, y que hay una relación mucho más fuerte entre la experiencia y la recomendación que entre la reputación y la recomendación.

Por tanto, podemos dar respuesta a la pregunta de investigación planteada, al cliente de los restaurantes de alto nivel le afecta la reputación en la experiencia y en la recomendación de este tipo de restaurantes, pero realmente disfruta de la experiencia y recomienda este tipo de restaurantes, no tanto por el prestigio que le proporciona, sino porque realmente le ha gustado y ha gozado de la experiencia.

Estos resultados pueden ser de gran utilidad para los gestores de los restaurantes, tanto para la ambientación y diseño del restaurante, como para la elaboración de la carta y los menús que componen la oferta gastronómica, así como la prestación del servicio en sí. Asimismo también pueden ser relevantes para la planificación de sus campañas de comunicación y a la hora de gestionar su presencia online tanto en el sitio web como en los perfiles de redes sociales. Sería en estos recursos donde se tendría que destacar el hecho de que comer en este tipo de restaurantes es una experiencia única.

Por otra parte también se podrían beneficiar los gestores de promoción de destinos o establecimientos turísticos en el que se puede ofrecer los servicios de estos restaurantes como una actividad complementaria dado que los consumidores lo consideran igual de importante que cualquier otra.

Una de las principales limitaciones es que la investigación se ha centrado en los restaurantes con estrella Michelin, y por tanto, no es aplicable a todo tipo de establecimientos culinarios. Asimismo, la encuesta hace referencia a restaurantes de un país en concreto, España, con lo que sería de interés ampliar el estudio a otras zonas geográficas para analizar la influencia de las variables culturales. En futuras líneas de investigación se podría profundizar en el análisis de los efectos directos e indirectos de los constructos analizados mediante modelos rivales. Una segunda línea de investigación trataría de averiguar cómo variables relativas a las características de los consumidores o de los restaurantes afectan a la recomendación, a la experiencia y a la reputación. Finalmente, otra línea futura de trabajo consistiría en investigar si la motivación de la experiencia gastronómica es lo suficientemente fuerte como para condicionar la selección del destino turístico.

\section{REFERENCIAS BIBLIOGRÁFICAS}

ADAM, I., ADONGO, C.A. y DAYOUR, F. (2015): «International Tourists' Satisfaction with Ghanaian Upscale Restaurant Services and Revisit Intentions», Journal of Quality Assurance in Hospitality \& Tourism, 16 (2), 181-201.

ANTÓN, C.; CAMARERO, C. y LAGUNA-GARCÍA, M. (2017): «Towards a new approach of destination loyalty drivers: satisfaction, visit intensity and tourist motivations», Current Issues in Tourism, 20 (3), 238-260.

BASRI, N.A.M. H., AHMAD, R., ANUAR, F.I. y ISMAIL, K.A. (2016): «Effect of Word of Mouth Communication on Consumer Purchase Decision: Malay Upscale Restaurant», Procedia-Social and Behavioral Sciences, 222, 324-331.

BJÖRK, P. y KAUPPINEN-RÄISÄNEN, H. (2017): «A destination's gastronomy as a means for holiday well-being», British Food Journal, 119 (7), 1578-1591. 
BOO, S.; BUSSER, J. y BALOGLU, S. (2009): «A model of custormer-based brand equity and its application to multiple destinations», Tourism Management, 30 (2), 219-231.

CARBALLO, R.; MORENO, S.; LEÓN, C. y BRENT, J.R. (2015): «La creación y promoción de experiencias en un destino turístico. Un análisis de la investigación y necesidades de actuación», Cuadernos de Turismo, 35, 71-94.

CARSTEN, M.K.; UHL-BIEN, M.; WEST, B.J.; PATERA, J.L. y MCGREGOR, R. (2010): «Exploring social constructions of followership: A qualitative study», The Leadership Quarterly, 21 (3), 543-562.

CHANG, R.C.Y., KIVELA, J., MAK, A.H.N., (2010). «Food preferences of Chinese tourists», Annals of Tourism Research, 37 (4), 989-1011

CHEN, C.F.; y CHEN, F.S. (2010): «Experience quality, perceived value, satisfaction and behavioral intentions for heritage tourists», Tourism Management, 31 (1), 29-35.

CHI, C.G. y QU, H. (2008): «Examining the structural relationships of destination image, tourist satisfaction and destination loyalty: An integrated approach», Tourism Management, 29 (4), 624-636.

CHIN, W.W. (1998): «Issues and opinion on structure equation modelling», MIS Quarterly, 22 (1), 7-161

COHEN, E. (1984): «The sociology of tourism: Approaches, issues and findings», Annual Review of Sociology, 10, 372-392.

COHEN, E y AVIELI, N. (2004): «Food in tourism: attraction and impediment», Annals of Tourism Research, 31 (4), 755-778

DARIES, N.; CRISTOBAL-FRANSI, E.; FERRER-ROSELL, B. y MARINE-ROIG, E. (2018): «Maturity and development of high-quality restaurant websites: A comparison of Michelin-starred restaurants in France, Italy and Spain», International Journal of Hospitality Management, 73, 125-137.

DARIES, N.; CRISTOBAL-FRANSI, E. y MARINE-ROIG, E. (2017): «Deployment of restaurants websites' marketing features: The case of Spanish Michelin-starred restaurants», International Journal of Hospitality \& Tourism Administration, Online press, 1-32.

EID, R. (2015): «Integrating Muslim Customer Perceived Value, Satisfaction, Loyalty and Retention in the Tourism Industry: An empirical study», International Journal of Tourism Research, 17 (3), 249-260.

FIELDS, K. (2002): «Demand for the gastronomy tourism product: motivational factors», in Hjalager, A., Richards, G. (Eds.), Tourism and Gastronomy. London, Routledge, 37-50.

FODNESS, D. (1994): «Measuring tourist motivation», Annals of Tourism Research, 21 (3), 555-581.

FORNELL, C. y LARCKER, D.F. (1981): «Structural equation models with unobservable variables and measurement error: algebra and statistics», Journal of Marketing Research, 18 (3), 382-388

GARCÍA HENCHE, B. (2017): «Los mercados de abastos y su comercialización como producto de turismo de experiencias. El caso de Madrid», Cuadernos de Turismo, 39, 167-189.

GONÇALVES, H.; DE FÁTIMA, M. y RITA, P. (2016): «Online purchase determinants of loyalty: The mediating effect of satisfaction in tourism», Journal of Retailing and Consumer Services, 30, 279-291. 
GOOLAUP, S.; SOLÉR, C. y NUNKOO, R. (2017). «Developing a Theory of Surprise from Travelers. Extraordinary Food Experiences», Journal of Travel Research. Online Press, 1-14.

GYIMÓTHY, S. y MYKLETUN, R.J. (2009): «Scary food: Commodifying culinary heritage as meal adventures in tourism», Journal of Vacation Marketing, 15 (3), 259-273.

HALL, J. y WINCHESTER, M., (2001): «Empirical analysis of Spawton's (1991) segmentation of the Australian wine market», Asia Pacific Advances in Consumer Research, 4, 319-327.

HJALAGER, A.M. (2004). «What do tourists eat and why? Towards a sociology of gastronomy and tourism», Tourism (Zagreb), 52 (2), 195-201.

HU, L.T. y BENTLER, P.M. (1999): «Cutoff criteria for fit indexes in covariance structure analysis: Conventional criteria versus new alternatives», Structural equation modelling: a multidisciplinary journal, 6, 1-55.

HYUN, S.S. y KANG, J. (2014): «A better investment in luxury restaurants: Environmental or non-environmental cues? », International Journal of Hospitality Management, 39, 57-70.

HYUN, S.S. y PARK, S. H. (2016): «The Antecedents and Consequences of Travelers» Need for Uniqueness: An Empirical Study of Restaurant Experiences», Asia Pacific Journal of Tourism Research, 21 (6), 596-623.

IGNATOV, E. y SMITH, S., (2006): «Segmenting Canadian culinary tourists», Current Issues in Tourism, 9 (3), 235-255.

JIMÉNEZ BELTRÁN, J.; LÓPEZ-GUZMÁN, T. y GONZÁLEZ SANTA-CRUZ, F. (2016): «Gastronomy and Tourism: Profile and Motivation of International Tourism in the City of Córdoba, Spain», Journal of Culinary Science \& Technology, 14 (4), 347-362.

KIM, J.H.; RITCHIE, J.R.B. y MCCORMICK, B. (2012): «Development of a Scale to Measure Memorable Tourism Experiences», Journal of Travel Research, 51 (1), 12-25.

KIM, Y.H.; KIM, M.; GOH, K.B. y ANTUN, M.J. (2011): «The role of money: the impact on food tourists' satisfaction and intention to revisit food events», Journal of Culinary Science \& Technology, 9 (2), 85-98.

KIM, Y.G.; EVES, A. y SCARLES, C. (2009): «Building a model of local food consumption on trips and holidays: A grounded theory approach», International Journal of Hospitality Management, 28 (3), 423-431.

KIM, Y. G.; SUH, B.W. y EVES, A. (2010): «The relationships between food-related personality traits, satisfaction, and loyalty among visitors attending food events and festivals», International Journal of Hospitality Management, 29 (2), 216-226

KIVELA, J. y CROTTS, J. (2006): «Tourism and gastronomy: gastronomy's influence on how tourists experience a destination», Journal of Hospitality and Tourism Research, 30 (3), 354-377.

KOZAK, M. y RIMMINGTON, M. (2000): «Tourist satisfaction with Mallorca, Spain, as an off-season holiday destination », Journal of Travel Research, 38 (1), 260-269.

KOZAK, M. (2001): «Repeaters' behavior at two distinct destinations», Annals of Tourism Research, 28 (3), 784-807.

LEE, W.H. y MOSCARDO, G. (2005): «Understanding the impact of ecotourism resort experiences on tourists $>$ environmental attitudes and behavioural intentions», Journal of Sustainable Tourism, 13 (6), 546-565. 
LIN, C.H. (2014): «Effects of cuisine experience, psychological well-being, and selfhealth perception on the revisit intention of hot springs tourists», Journal of Hospitality \& Tourism Research, 38 (2), 243-265.

LIVINGSTONE, S. (2008): «Taking risky opportunities in youthful content creation: teenagers' use of social networking sites for intimacy, privacy and self-expression», New Media \& Society, 10 (3): 393-411.

LONG, L.M. (Ed.) (2004): Culinary tourism. Kentucky, The University Press of Kentucky. MAK, A. H., LUMBERS, M., EVES, A. y CHANG, R.C. (2013): «An application of the repertory grid method and generalised Procrustes analysis to investigate the motivational factors of tourist food consumption», International Journal of Hospitality Management, 35, 327-338.

MCINTOSH, R.; GOELDNER, C. y RITCHIE, J. (1995): Tourism Principles, Practices, Philosophies, 7th ed. New York, Wiley.

MEDINA, F.X. (2015): «Turismo y Cultura en Denominaciones de Origen Enogastronómicas: El Caso de la Región de Tokaj-Hegyalja (Hungría)», International Journal of Scientif Management and Tourism, 3, 167-178.

MILLÁN VÁZQUEZ DE LATORRE, G.; HERNÁNDEZ ROJAS, R. y NAVAJAS ROMERO, V. (2016): «The study of gastronomic tourism in Cordoba and the association of the cuisine. An econometric analysis», Tourism and Hospitality Management, 22 (2), 173-191.

OLIVEIRA, S. (2011): «La gastronomía como atractivo turístico primario de un destino: El Turismo Gastronómico en Mealhada-Portugal», Estudios y Perspectivas en Turismo, 20 (3), 738-752.

OTIS, L.P. (1984): «Factors influencing the willingness to taste unusual foods», Psychological Report, 54, 739-745

POLLARD, J.; KIRK, S.F. y CADE, J.E. (2002): «Factors affecting food choice in relation to fruit and vegetable intake: a review», Nutrition Research Reviews, 15, 373-387.

QUAN, S. y WANG, N. (2004): «Towards a structural model of the tourist experience: An illustration from food experiences in tourism», Tourism management, 25 (3), 297-305.

RIVERA, M. (2013): «El turismo experiencial como forma de turismo responsable e intercultural», Relaciones interculturales en la diversidad, 2 (1), 199-217.

RUST, R.T. y OLIVER, R.L. (2000): «Should we delight the customer? », Journal of the Academy of Marketing Science, 28 (1), 86-94.

SPARKS, B.; BOWEN, J. y KLAG, S. (2003): «Restaurant and the tourist market», International Journal of Contemporary Hospitality Management, 15 (1), 6-13.

SUAU, F. (2012): «El turista 2.0 como receptor de la promoción turística: estrategias lingüísticas e importancia de su estudio», Revista Pasos de Turismo y Patrimonio Cultural, 10 (4), 143-153.

THOMPSON, M. y PRIDEAUX, B. (2009): «Developing a food and wine segmentation and classifying destinations on the basis of their food and wine sectors», Advances in hospitality and leisure, 5, pp. 163-183.

TSAI, C.T. y LU, P.H. (2012): «Authentic dining experiences in ethnic theme restaurants», International Journal of Hospitality Management, 31 (1), 304-306.

TSAI, C.T.S. y WANG, Y. C. (2017): «Experiential value in branding food tourism», Journal of Destination Marketing \& Management, 6 (1), 56-65. 
TUNG, V.W.S. y RITCHIE, J.R.B. (2011): «Exploring the essence of memorable tourism experiences», Annals of Tourism Research, 38 (4), 1.367-1.386.

VAN DER HEIJDEN, H. (2004): «User acceptance of hedonic information systems», MIS quarterly, 695-704.

WAKEFIELD, R. y WAKEFIELD, K. (2016): «Social media network behavior: A study of user passion and affect », The Journal of Strategic Information Systems, 25 (2), 140-156.

WARDE, A. y MARTENS, L. (2000): Eating Out: Social Differentiation, Consumption and Pleasure. Cambridge, Cambridge University Press.

WOLF, M.M.; BERTOLINI, P. y PARKER-GARCIA, J. (2004): «A Comparison of Consumer Attitudes towards GM Food in Italy and the USA», Consumer Acceptance of Genetically Modified Foods, 131.

YANG, W. y MATTILA, A. (2014): «Do affluent customers care when luxury brands go mass?: The role of product type and status seeking on luxury brand attitude», International Journal of Contemporary Hospitality Management, 26 (4), 526-543.

YANG, W. y MATTILA, A. (2017): «The Impact of Status Seeking on Consumers' Word of Mouth and Product Preference: A Comparison Between Luxury Hospitality Services and Luxury Goods », Journal of Hospitality \& Tourism Research, 41 (1), 3-22.

YOON, Y. y UYSAL, M. (2005): «An examination of the effects of motivation and satisfaction on destination loyalty: A structural model», Tourism Management, 26 (1), 45-56.

ZHENG, X. y GRETZEL, U. (2010): «Role of social media in online travel information search», Tourism Management, 31 (2), 179-188. 